\title{
Antibiotic resistance and biofilm formation among coagulase-negative staphylococci isolated from clinical samples at a tertiary care hospital of eastern Nepal
}

\author{
Lok Bahadur Shrestha*, Narayan Raj Bhattarai and Basudha Khanal
}

\begin{abstract}
Background: Coagulase negative staphylococci were long regarded non-pathogenic as they are the commensals of human skin and mucosa but the recent changes in the medical practice and changes in underlying host populations, they are being considered significant pathogens associated with number of nosocomial infections. The objective of the study was to determine the species, antimicrobial susceptibility pattern, biofilm forming ability of the clinically significant CoNS isolates and to compare the different methods for the detection of biofilm formation.

Methods: A total of 52 clinically significant CoNS isolates obtained from different units during a year period were studied. Characterization was done using standard microbiological guidelines and antimicrobial susceptibility was done following CLSI guidelines. Biofilm formation was detected by using three methods i.e. tissue culture plate method, congo red agar method and tube adherence method.

Results: Among 52 isolates, S. epidermidis (52\%) was the most common species which was followed by S. saprophyticus (18\%) and S. haemolyticus (14\%). Antimicrobial susceptibility pattern of CoNS documented resistance of $80 \%$ to ampicillin. Resistance to cefoxitin and ceftriaxone was observed in $58 \%$ of the isolates. Biofilm formation was observed in $65.38 \%$ of the isolates. The accuracy of Congo red agar and tube adherence method for the detection of biofilm formation was $82 \%$ and $76 \%$ respectively.

Conclusion: CoNS isolates obtained from clinical samples should be processed routinely and antimicrobial susceptibility testing should be performed. Multidrug-resistant CoNS are prevalent. All the three methods i.e. tissue culture plate, Congo red agar and tube adherence method can be used in detecting biofilm formation.
\end{abstract}

Keywords: CoNS, Nosocomial infection, Biofilm

\section{Background}

Coagulase-negative staphylococci (CoNS) are the most abundant normal microbial flora of the human skin and mucous membranes. They were long regarded nonpathogenic as they are the commensals of human skin and mucosa. Due to the recent changes in the medical practice and changes in underlying host populations, they are being considered significant pathogens associated with a number of nosocomial infections $[1,2]$.

\footnotetext{
* Correspondence: lok.shrestha@bpkihs.edu; drlok86@gmail.com Microbiology \& Infectious Diseases, B. P. Koirala Institute of Health Sciences (BPKIHS), Dharan, Nepal
}

CoNS is now considered one of the most common causes of the device related infections in the recent two decades, especially in immunocompromised patients and the chronic debilitated patient who need the long-term central venous access [3]. They may adhere to medical devices and surfaces through slime layer which has a muco-polysaccharide structure, thus they may easily colonize and spread within the hospital environment. The slime factor also aids in pathogenicity by protecting them from phagocytosis, chemotaxis and also antimicrobial agents [4]. Studies suggest that selective advantage for CoNS in causing device associated infections is 
achieved by its ability to adhere to plastic catheters more aggressively than any other organism [5].

CoNS isolated from nosocomial environments are usually resistant to multiple antimicrobial agents. About 80\%-90\% of CoNS isolates associated with hospital infections are methicillin resistant coagulase-negative staphylococci (MRCoNS) [4].

With the advancement of medical sciences, especially with the increasing use of medical devices, the infections caused by CoNS are ever increasing. The characterization of CoNS upto species level, their antimicrobial properties and biofilm production is very important for its diagnosis and treatment. The present study is therefore proposed to determine the species distribution, antimicrobial susceptibility and surface adherence property of CoNS isolated at BPKIHS hospital.

\section{Methods}

Isolates

The study was carried out in the Department of Microbiology, B.P. Koirala Institute of Health Sciences, Dharan, Nepal from July 2013 to June 2014. Consecutive sampling method was used i.e. all the clinically significant CoNS isolates obtained during the study period were enrolled. A total of $52 \mathrm{CoNS}$ isolates were obtained from various clinical specimens (blood, pus, urine, CVC, tracheostomy tube, tissue) were included in the study.

\section{Identification and characterization of CoNS}

Bacterial isolates obtained were identified by colony morphology, gram staining and biochemical tests (catalase test, slide coagulase, tube coagulase and mannitol test). Once the identity of the isolate as CoNS was confirmed, several biochemical tests and antimicrobial discs were used for their characterization upto species level. All the tests were performed according to standard microbiological methodology [6, 7].

\section{Antimicrobial susceptibility test: $[8,9]$}

Antimicrobial susceptibility testing of the isolates was performed on Mueller-Hinton Agar by.

Kirby-Bauer disk diffusion method recommended by clinical laboratory standard institution.

(CLSI) guidelines against following antimicrobial discs (HiMedia Laboratories): amikacin, ampicillin, ceftriaxone, ciprofloxacin, azithromycin, cotrimoxazole, vancomycin and linezolid.

Cefoxitin disc was used to detect methicillin resistance. Staphylococcus aureus ATCC 25923 and Escherichia coli ATCC 25922 were used as control strains and tested along with the test strains.
Determination of minimum inhibitory concentration (MIC) [10]

MIC of oxacillin and Vancomycin was determined by agar dilution technique as per CLSI guidelines. Oxacillin powder $(815 \mu \mathrm{g} / \mathrm{g})$ and Vancomycin powder (potency $=930 \mu \mathrm{g} / \mathrm{g}$ ) was obtained from HiMedia laboratories India. The concentration at which complete inhibition of growth is achieved is considered the MIC. The results were interpreted according to the CLSI guidelines.

\section{Study of biofilm formation}

Biofilm production were investigated by the tube adherence test described by Christensen et al. (1982) and Congo red agar method as described by Freeman et al. (1989) and Tissue culture plate method as described by Christensen et al. (1985) $[5,11,12]$. Results were interpreted considering Tissue culture plate method as gold standard [13-15].

\section{Tube adherence method [5]}

A loopful of bacterial suspension from overnight culture were inoculated into the Trypticase.

Soy Broth with $1 \%$ glucose (TSBGlu, $10 \mathrm{~mL}$ ) and incubated for $24 \mathrm{~h}$ at $37{ }^{\circ} \mathrm{C}$. The tubes were gradually poured down and was washed with phosphate buffer solution ( $\mathrm{pH}$ 7.3). After drying, the tubes were stained with $0.1 \%$ crystal violet. Excess stains were removed and tubes were washed away with water multiple times. Tubes were then kept in inverted position and finally observed for biofilm formation.

\section{Congo red agar method [11]}

Congo red agar was prepared by mixing $37 \mathrm{~g}$ Brain heart infusion broth (HiMedia), $50 \mathrm{~g}$ sucrose (HiMedia), $0.8 \mathrm{~g}$ congo red dye and $10 \mathrm{~g}$ agar in $1 \mathrm{l}$ distilled water. The solution was then sterilized by autoclave $\left(121{ }^{\circ} \mathrm{C}, 15 \mathrm{lbs}\right.$. for $15 \mathrm{~min}$ ) and dispensed onto $90 \mathrm{~mm}$ petri dishes. CoNS isolates were plated and were incubated at $37{ }^{\circ} \mathrm{C}$ for $24 \mathrm{~h}$. Biofilm production was detected by culturing the CoNS isolates on congo red agar plates. The biofilm forming strains produced black colonies while non-forming strains developed red colonies.

\section{Tissue culture plate (TCP) method [12]}

Trypticase soy broth with $1 \%$ glucose (TSBglu) was used as culture media. Isolates from fresh agar plates were inoculated in TSBglu media and incubated for 18-24 h at $37{ }^{\circ} \mathrm{C}$ in aerobic condition. After incubation, $0.2 \mathrm{ml}$ of culture was filled onto individual wells of 96 well flatbottom tissue culture plates. For control to check sterility and non-specific binding of media, only broth without the bacteria was used. The tissue culture plates were incubated aerobically at $37{ }^{\circ} \mathrm{C}$ for $18-25 \mathrm{~h}$. The plates were gently tapped for the removal of unbound content. 


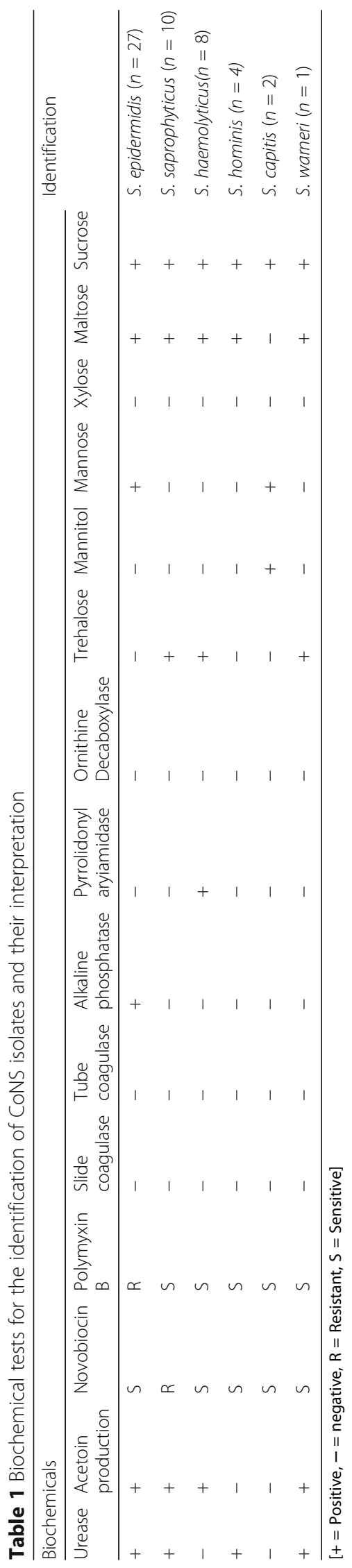




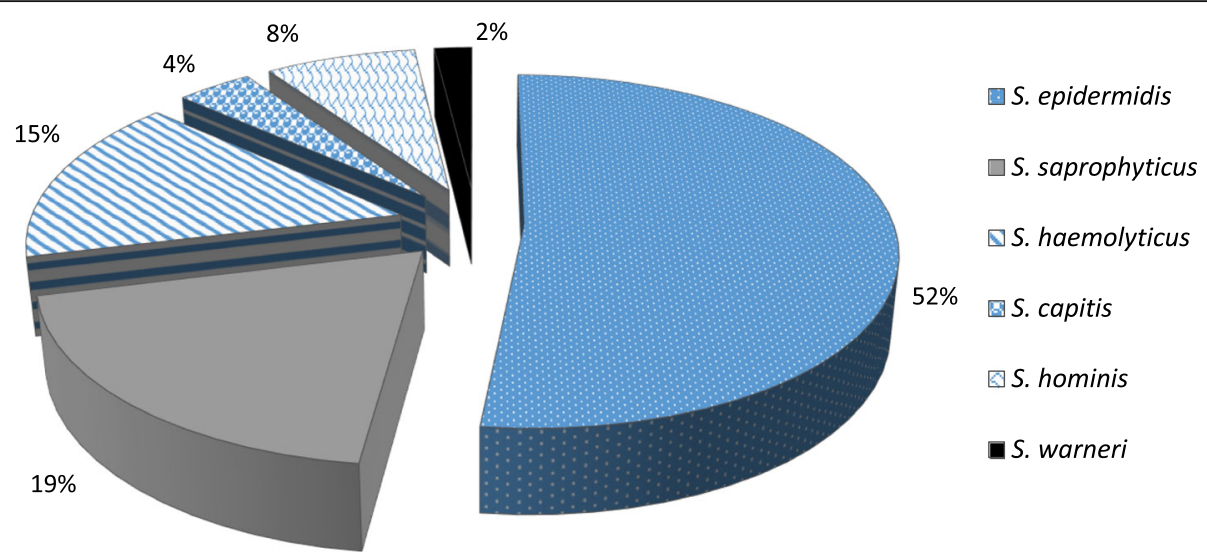

Fig. 1 Different species of coagulase-negative staphylococci $(n=52)$

For the removal of free floating bacteria, the individual wells were cleaned four times with $0.2 \mathrm{ml}$ of phosphate buffer saline (PBS pH 7.2). The biofilms formed by adherent organisms in tissue culture plate were fixed with sodium acetate $(2 \%)$ and stained with $0.1 \%$ crystal violet. The plates were washed with water for excess stain and were left for drying. The isolates which formed biofilm were adherent on all side wells and stained uniformly with crystal violet.

\section{Results}

A total of 52 CoNS isolates were studied over the duration of 1 year (July 2013-june 2014). The species identification was done on the basis of standard guidelines $[6,7]$ using the biochemical tests which is interpreted in Table 1. Among them, S. epidermidis (52\%) was the most common species, followed by $S$. saprophyticus (18\%) and S. haemolyticus (14\%). The overall species distribution is further elicitated on Fig. 1.
Antimicrobial susceptibility pattern of CoNS documented resistance of $80 \%$ of isolates to ampicillin. Resistance to ceftriaxone was observed in $58 \%$ of the isolates. Among 52 isolates,

Twenty two were susceptible to methicillin as indicated by cefoxitin disc diffusion $(\leq 24 \mathrm{~mm})$ and 30 were resistant. Methicillin resistance was confirmed by MIC of oxacillin against these isolates. All the isolates were susceptible to vancomycin and linezolid. Susceptibility to vancomycin was confirmed by calculating MIC of vancomycin against these isolates (Fig. 2).

The present study also showed that MRCoNS are comparatively more resistant to multiple antimicrobial agents. Isolates obtained from critical care units were more resistant strains as compared to isolates obtained from the outpatient department and wards.

\section{Study of biofilm formation}

Biofilm formation was studied in this study by three methods i.e. Tissue culture plate method,

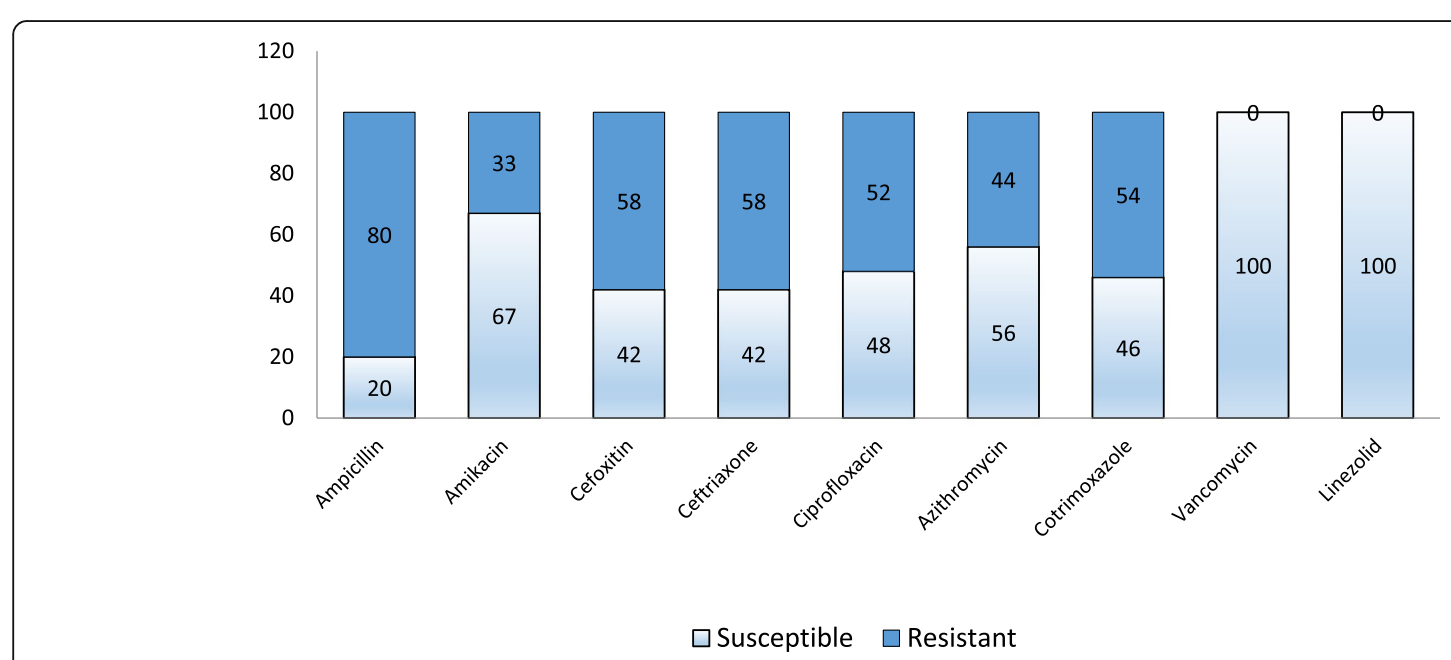

Fig. 2 Antimicrobial susceptibility pattern of different CoNS isolates against several antimicrobial agents (\%) 
Table 2 Biofilm formation by Congo red agar, tube adherence and tissue culture plate methods

\begin{tabular}{llll}
\hline & & \multicolumn{2}{l}{ Tissue culture plate method } \\
\cline { 3 - 4 } & & Positive & Negative \\
\hline Congo red method & Positive & 28 & 1 \\
& Negative & 6 & 17 \\
\multirow{2}{*}{ Tube adherence method } & Positive & 26 & 1 \\
& Negative & 8 & 17 \\
\hline
\end{tabular}

Congo red agar method and tube adherence method. Among 52 isolates, 34 isolates were biofilm formers; 34 of them were detected by tissue culture plate method, 29 by congo red agar method while 27 isolates were biofilm positive by tube adherence method.

The tissue culture plate method was used as the standard method to evaluate the congo red agar and tube adherence method [13-15]. Sensitivity, specificity, positive predictive value, negative predictive value, and accuracy were calculated by analysis of $2 * 2$ table (Table 2). The sensitivity of congo red agar was $82.35 \%$ which is higher than tube adherence method $76.47 \%$. The specificity of two tests was similar 94\%. The accuracy of congo red agar method $(86.5 \%)$ was also higher than tube adherence method (82.69\%) (Table 3).

Biofilm formation was seen most commonly in S. epidermidis followed by $S$. haemolyticus.

The biofilm producers were resistant to multiple antibiotics in comparison to biofilm non-formers. Resistance to ampicillin and methicillin was seen in $100 \%$ and $86 \%$ of biofilm producers while only $42 \%$ and $30 \%$ resistance was seen in biofilm non-producers (Fig. 3).

The biofilm producer isolates were studied on the basis of the origin of the sample. All the isolates obtained from the intensive care unit (ICU) were biofilm former while no isolates obtained from outpatient department produced biofilm.

\section{Discussion}

\section{General distribution and speciation}

A total of 52 clinically significant isolates of coagulasenegative staphylococci obtained during the period of 1 year (July 2013-June 2014) were included in our study. Among the isolates, 6 different species of coagulase-negative staphylococci were encountered: S. epidermidis (27, 52\%), S. saprophyticus (10, 19\%), S. haemolyticus (8, 15\%), S. hominis $(4,8 \%)$, S. capitis $(2,4 \%)$ and S. warneri $(1,2 \%)$. The findings of the present study are in agreement with the various studies which shows $S$. epidermidis as the most common coagulase-negative staphylococci $[16,17]$. In contrast to the finding of our study, Jain A et al. isolated S. haemolyticus as (58\%) as the most common isolate, followed by $S$. epidermidis (17\%) [18]. S. epidermidis was isolated from various clinical specimens like blood, central venous catheter, pus and urine, while $S$. saprophyticus was predominantly obtained from urine samples (90\%). The result correlates with the other studies which reported the pathogenic role of $S$. epidermidis in catheter related sepsis and that of S. saprophyticus in UTI $[4,5]$. The higher incidence of $S$. saprophyticus in UTI is due to its colonization of the rectum or urogenital tracts of $5-10 \%$ of women [4].

\section{Antimicrobial resistance}

In our study, the antimicrobial susceptibility of isolates was tested against 9 antimicrobials in accordance with the CLSI guidelines [8]. AST pattern of the isolates showed $80 \%$ resistance to ampicillin, $55 \%$ to ceftriaxone. Fifty-eight percent of the isolates were characterized as methicillin-resistant CoNS. All the 52 isolates were susceptible to vancomycin and linezolid. The susceptibility of vancomycin was confirmed by MIC against the isolates. The result of our study is consistent with other research article [19]. However some studies have suggested that vancomycin resistance does exist and is in increasing trends. Natoli $\mathrm{S}$ et al. observed no resistance to vancomycin but $5.4 \%$ isolates had reduced susceptibility to vancomycin [20].

Isolates obtained from intensive care unit (ICU + NICU) were more resistant when compared to other wards and outpatient department. A similar pattern of drug resistance was documented by several other studies $[19,21]$. The patients hospitalized in intensive care unit are managed with multiple indwelling medical devices for the medication, nutrition and respiration. They are more vulnerable to biofilm formation and subsequent infection [22].

\section{Biofilm formation}

Among the 52 isolates, 34 (65.38\%) isolates were biofilm formers. Biofilm formation was detected by tissue culture plate method in 34 isolates, congo red agar in 29 isolates, while 27 isolates showed positive biofilm formation test by tube adherence method. Similar results were obtained in previous studies $[23,24]$.

Table 3 Statistical evaluation of Congo red agar and Tube adherence method for detection of biofilm Im formation in staphylococci using the tissue culture plate method as the standard method

\begin{tabular}{llllll}
\hline & Sensitivity & Specificity & PPV & NPV & Accuracy \\
\hline Congo red agar & 82.35 & 94.5 & 96.5 & 43.47 & 86.5 \\
Tube adherence method & 76.47 & 94.44 & 96.29 & 68 & 82.69 \\
\hline
\end{tabular}




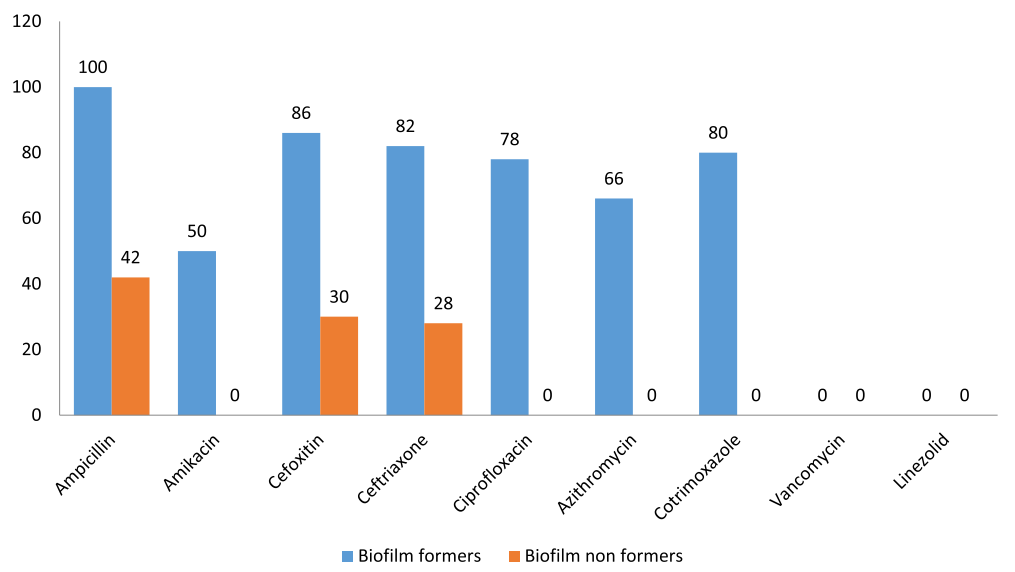

Fig. 3 Antimicrobial resistance pattern with relation to biofilm formation property

Congo red agar detected 85\% (29/34) biofilm producer and tube adherence method detected 79\% (27/34). Tissue culture plate is used as the standard method for detection of biofilm formation [13-15] and the results of our study supports the evidence. Similar results were documented in various studies $[13,17]$.

The sensitivity, specificity, accuracy of the two tests were calculated using tissue culture plate as the reference [13-15]. The sensitivity (82.35\%) and accuracy $(86.5 \%)$ of congo red agar were higher than tube adherence method (sensitivity $=76.47$, accuracy $=82.69 \%$ ), while no difference was observed in the specificity of these two methods. The positive predictive value of both congo red agar and tube adherence method is good (above 90), and they may therefore be used in clinical decision making. Similar results were documented by Saising et al. [24].

The present study also reveals that $100 \%$ of isolates obtained from intensive care units were biofilm formers while only $40 \%$ of isolates from surgery ward and none of the isolates obtained from the outpatient departments were biofilm formers. Similarly, the biofilm producers were more resistant to antimicrobial agents as compared to nonproducers which agree with the study done in the past [5].

\section{Conclusion}

A total of 52 isolates of CoNS were studied, among which 6 species were identified. S. epidermidis (52\%) was the most common species followed by $S$ saprophyticus (19\%), S. haemolyticus (15\%) and S. hominis (8\%). Antimicrobial susceptibility pattern of CoNS documented resistance of $80 \%, 58 \%$ and $58 \%$ resistance to ampicillin, ceftriaxone and cefoxitin respectively. All the isolates were susceptible to vancomycin and linezolid. Biofilm production was detected in $67 \%$ of the isolates. Tissue culture plate is the most effective method for the detection of biofilm formation while congo red agar and tube adherence method can also detect biofilm formation with an accuracy of $86 \%$ and $82 \%$ respectively.

\section{Abbreviations}

AMR: Antimicrobial resistance; ATCC: American type culture collection; CLSI: Clinical and laboratory standards institute; CoNS: Coagulase-negative Staphylococci; CVC: Central vascular catheter; FDA: Food and Drug Administration; HIV: Human immunodeficiency virus; ICU: Intensive Care Unit; MIC: Minimum Inhibitory Concentration; MRCoNS: Methicillin-resistant Coagulse-negative Staphylococci; MSCoNS: Methicillin-sensitive Coagulasenegative Staphylococci; NICU: Neonatal intensive care unit; UTI: Urinary tract infection

Acknowledgements

All members of the Department of Microbiology.

Funding

None.

Availability of data and materials

Yes, available.

Authors' contributions

Conceptualization: LBS, NRB, BK. Investigation: LBS. Methodology: LBS, NRB, BK. Resources: LBS, NRB, BK. Supervision: NRB, BK. Writing-original draft: LBS. Writing-review and editing: NRB, BK. All authors read and approved the final manuscript.

\section{Ethics approval and consent to participate}

$$
\begin{aligned}
& \text {-was obtained from Institutional Ethical Review Board (IERB) } \\
& \text {-code no IERB/196/014 } \\
& \text {-consent to participate: not applicable }
\end{aligned}
$$

\section{Consent for publication}

Not applicable.

\section{Competing interests}

The authors declare that they have no competing interests.

\section{Publisher's Note}

Springer Nature remains neutral with regard to jurisdictional claims in published maps and institutional affiliations. 
Received: 27 April 2017 Accepted: 25 August 2017

Published online: 31 August 2017

\section{References}

1. Rupp ME, Archer GL. Coagulase-negative staphylococci: pathogens associated with medical progress. Clin Infect Dis. 1994;19(2):231-43. quiz 445. [PMID: 7986894]

2. Bannerman TL. Staphylococcus, micrococcus, and other catalasepositive cocci that grow aerobically. In: Murray PR, Baron EJ, Jorgensen JH, editors. Manual of medical microbiology. Washington, DC: American Society For Microbiology; 2003. p. 384-404

3. Schulin T, Voss A. Coagulase-negative staphylococci as a cause of infections related to intravascular prosthetic devices: limitations of present therapy. Clin Microbiol Infect. 2001;7(Suppl 4):1-7. [PMID: 11688530]

4. Rupp ME, Fey PD. Staphylococcus Epidermidis and other Coagulase negative staphylococci. In: Mandell GL, Benett JE, Dolin R, editors. Mandell, Douglas and Bennett's principles and practice of infectious disease. 7th ed Philadelphia: Churchill Livingstone Elsevier; 2010. p. 186.

5. Christensen GD, Simpson WA, Bisno AL, Beachey EH. Adherence of slimeproducing strains of Staphylococcus Epidermidis to smooth surfaces. Infect Immun. 1982;37(1):318-26. [PMID: 6179880]

6. Kloos WE, Schleifer $\mathrm{KH}$. Simplified scheme for routine identification of human staphylococcus species. J Clin Microbiol. 1975;1(1):82-8. PMID: 170303

7. Winn W, Allen S, Janda W. Koneman's color atlas and textbook of diagnostic microbiology. 6th ed. Philadelphia: Lippincott Williams and Wikins; 2006. p. 623-62.

8. Clinical and Laboratory Standard Institute. Performance standard for antimicrobial disk susceptibility tests, Document no M100-S23. 23rd ed. Wayne: Clinical and Laboratory Standard Institute; 2013.

9. Bauer AW, Kirby WMM, Sherris JC, Turck M. Antibiotic susceptibility testing by a standardized single disc method. Am J Clin Pathol. 1966;45:493.

10. Clinical and Laboratory Standards Institute. Methods for dilution antimicrobial susceptibility tests for bacteria that grow aerobically, CLSI document no M07-A9. 9th ed. Wayne: Clinical and Laboratory Standards Institute; 2012.

11. Freeman DJ, Falkiner FR, Keane CT. New method for detecting slime production by coagulase negative staphylococci. J Clin Pathol. 1989;42(8): 872-4. PMID: 2475530

12. Christensen GD, Simpson WA, Younger JJ, Baddour LM, Barrett FF, Melton DM, et al. Adherence of coagulase-negative staphylococci to plastic tissue culture plates: a quantitative model for the adherence of staphylococci to medical devices. J Clin Microbiol. 1985;22(6):996-1006. PMID: 3905855

13. Mathur T, Singhal S, Khan S, Upadhyay DJ, Fatma T, Rattan A. Detection of biofilm formation among the clinical isolates of staphylococci: an evaluation of three different screening methods. Indian J Med Microbiol. 2006;24(1):259. PMID: 16505551

14. Panda PS, Chaudhary U, Dube SK. Comparison of four different methods for detection of biofilm formation by uropathogens. Indian J Pathol Microbiol. 2016:59(2):177-9. PMID: 27166035

15. Hassan A, Usman J, Kaleem F, Omair M, Khalid A, lqbal M. Evaluation of different detection methods of biofilm formation in the clinical isolates. Braz J Infect Dis. 2011;15(4):305-11.

16. Ferreira RB, lorio NL, Malvar KL, Nunes AP, Fonseca LS, Bastos CC, et al. Coagulase-negative staphylococci: comparison of phenotypic and genotypic oxacillin susceptibility tests and evaluation of the agar screening test by using different concentrations of oxacillin. J Clin Microbiol. 2003; 41(8):3609-14. PMID: 12904363

17. Alcaraz LE, Satorres SE, Lucero RM, Centorbi OM. Species identification, slime production and oxacillin susceptibility in coagulase-negative staphylococci isolated from nosocomial specimens. Braz J Microbiol. 2003;34:45-1.

18. Jain A, Agarwal J, Bansal S. Prevalence of methicillin-resistant, coagulase-negative staphylococci in neonatal intensive care units: findings from a tertiary care hospital in India. J Med Microbiol. 2004; 53(Pt 9):941-4. PMID: 15314204

19. Jarlov JO. Phenotypic characteristics of coagulase-negative staphylococci: typing and antibiotic susceptibility. APMIS Suppl. 1999;91: 1-42. PMID: 10230367

20. Natoli S, Fontana C, Favaro M, Bergamini A, Testore GP, Minelli S, et al. Characterization of coagulase-negative staphylococcal isolates from blood with reduced susceptibility to glycopeptides and therapeutic options. BMC Infect Dis. 2009;9:83. PMID: 19497104
21. Zhao C, Sun H, Wang H, Liu Y, Hu B, Yu Y, et al. Antimicrobial resistance trends among 5608 clinical gram-positive isolates in China: results from the gram-positive Cocci resistance surveillance program (2005-2010). Diagn Microbiol Infect Dis. 2012;73(2):174-81.

22. Pfaller MA, Herwaldt LA. Laboratory, clinical, and epidemiological aspects of coagulase-negative staphylococci. Clin Microbiol Rev. 1988;1(3):281-99. PMID: 3058297

23. Karthik S, Bhattacharya S, Harish BN, Parija SC. Detection of slime production by coagulase-negative staphylococcus: an assessment of two methods. Indian J Pathol Microbiol. 2004;47(1):85-9. PMID: 15471145

24. Saising J, Singdam S, Ongsakul M, Voravuthikunchai SP. Lipase, protease, and biofilm as the major virulence factors in staphylococci isolated from acne lesions. Biosci Trends. 2012;6(4):160-4. PMID: 23006962

\section{Submit your next manuscript to BioMed Central and we will help you at every step:}

- We accept pre-submission inquiries

- Our selector tool helps you to find the most relevant journal

- We provide round the clock customer support

- Convenient online submission

- Thorough peer review

- Inclusion in PubMed and all major indexing services

- Maximum visibility for your research

Submit your manuscript at www.biomedcentral.com/submit
) Biomed Central 